\title{
ISO observations of low and moderate albedo asteroids
}

\section{PHT-P and PHT-S results ${ }^{\star}$}

\author{
E. Dotto ${ }^{1,2}$, M. A. Barucci ${ }^{3}$, T. G. Müller ${ }^{4,5}$, J. R. Brucato ${ }^{6}$, M. Fulchignoni ${ }^{2,7}$, \\ V. Mennella ${ }^{6}$, and L. Colangeli ${ }^{6}$
}

1 INAF - Osservatorio Astronomico di Torino, Strada Osservatorio 20, 10025 Pino Torinese (TO), Italy

2 INAF - Osservatorio Astronomico di Roma, via Frascati 33, 00040 Monteporzio Catone (Roma), Italy

3 LESIA - Observatoire de Paris, 5 place J. Jannsen, 92195 Meudon Principal Cedex, France e-mail: antonella.barucci@obspm.fr

${ }^{4}$ Max-Planck-Institut fuer extraterrestrische Physik, Giessenbachstrasse, 85748 Garching, Germany e-mail: tmueller@mpe.mpg.de

5 Data Centre, ESA, Villafranca Del Castillo, Apdo 50727, 28080 Madrid, Spain (until Dec. 2001)

${ }^{6}$ INAF - Osservatorio Astronomico di Capodimonte, via Moiariello 16, 80131 Napoli, Italy e-mail: brucato@na.astro.it; mennella@na.astro.it; colangeli@na.astro.it

7 Université Paris VII, Paris, France e-mail: marcello.fulchignoni@obspm.fr

Received 19 April 2002 / Accepted 20 June 2002

\begin{abstract}
The ISO observations presented here are devoted to low and moderate albedo asteroids, which are supposed to be among the more primitive objects of the solar system. Spectroscopic and multi-filter photometric data of 77 Frigga, 114 Kassandra, 308 Polyxo, 511 Davida, and 914 Palisana have been obtained by the ISO instrument ISOPHOT. The subsystem PHT-P carried out photometric observations at 10, 12, 25, and $60 \mu \mathrm{m}$, while low resolution spectra have been acquired by the subsystem PHT-S between 5.8 and $11.6 \mu \mathrm{m}$. The Standard Thermal Model and a black-body fit have been applied to the obtained data in order to model the thermal continuum and to derive sub-solar and black-body temperatures. To interpret the obtained results and to investigate the surface composition of the observed asteroids, we compare the ISO spectra with the whole sample of mineral and meteorite laboratory spectra available in the literature. New laboratory experiments performed at the Capodimonte Observatory have been carried out to increase the available sample. A tentative spectral similarity with meteorites is presented.
\end{abstract}

Key words. minor planets, asteroids - infrared: solar system

\section{Introduction}

Low albedo asteroids are classified in the current taxonomies (Tholen \& Barucci 1989) as belonging to three different classes: C, P, and D. They are believed to be formed in the outer part of the main belt, to have been less thermally processed and to have preserved primordial materials on their surface. High albedo asteroids are supposed to have been formed at small heliocentric distances and to have been more thermally evolved. As shown by several authors, asteroids in the main belt

Send offprint requests to: E. Dotto, e-mail: dotto@to.astro.it

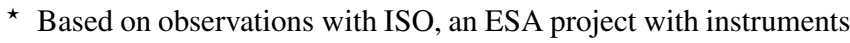
funded by ESA Member States (especially the PI countries: France, Germany, the Netherlands and the UK) with the participation of ISAS and NASA. display a systematic variation of spectral classes with heliocentric distance (e.g. Gradie et al. 1989). The decline in hydrated silicate abundance with increasing heliocentric distance seems to be due to the fact that hydrated silicates are more thermally processed than pristine materials. Jones et al. (1990) proposed a scenario for the formation of low albedo asteroids: accretion of ice and anhydrous silicates from the solar nebula combined with relic interstellar organic kerogen, formed very dark, volatile-rich asteroids, of which P and D-types may be representative. Selective induction heating produces aqueous alteration at the surface. This is the low temperature chemical alteration of materials by liquid water which acts as a solvent and produces materials like phyllosilicates, oxides, sulfates, carbonates and hydroxides. The same selective induction heating perhaps produced in other cases melting in the interior. 
Table 1. Aspect data of the ISO observations: Target Dedicated Time (TDT) number of the observation, detector, observation date and mid time, heliocentric distance $r$, geocentric distance $\Delta$, phase angle $\alpha$, and solar elongation $\lambda-\lambda_{\odot}$.

\begin{tabular}{|c|c|c|c|c|c|c|c|c|c|}
\hline Object & & $\begin{array}{c}\text { TDT } \\
\text { number }\end{array}$ & Detector & $\begin{array}{c}\text { Observation } \\
\text { Date }\end{array}$ & $\begin{array}{l}\text { Obs. mid time } \\
\text { (UT) }\end{array}$ & $\begin{array}{c}r \\
{[\mathrm{AU}]}\end{array}$ & $\begin{array}{c}\Delta \\
{[\mathrm{AU}]}\end{array}$ & $\begin{array}{c}\alpha \\
{[\mathrm{deg}]}\end{array}$ & $\begin{array}{c}\lambda-\lambda_{\odot} \\
{[\mathrm{deg}]}\end{array}$ \\
\hline \multirow[t]{4}{*}{ (77) Frigga } & on & 53500120 & PHT-P & 04-May-1997 & $02: 30$ & 2.7174 & 2.9556 & 19.9 & 66.6 \\
\hline & on & 53500121 & PHT-S & 04-Мay-1997 & $02: 45$ & & & & \\
\hline & off & 53700122 & PHT-P & 06-Мay-1997 & $02: 21$ & & & & \\
\hline & off & 53700123 & PHT-S & 06-Мay-1997 & $02: 37$ & & & & \\
\hline \multirow[t]{4}{*}{ (114) Kassandra } & on & 62500106 & PHT-P & 01-Aug.-1997 & $21: 30$ & 2.6388 & 2.3271 & 22.5 & 96.3 \\
\hline & on & 62500107 & PHT-S & 01-Aug.-1997 & $21: 45$ & & & & \\
\hline & off & 62700108 & PHT-P & 03-Aug.-1997 & $21: 23$ & & & & \\
\hline & off & 62700109 & PHT-S & 03-Aug.-1997 & $21: 38$ & & & & \\
\hline \multirow[t]{4}{*}{ (308) Polyxo } & on & 51600113 & PHT-P & 15-Apr.-1997 & $03: 44$ & 2.6455 & 2.6344 & 21.9 & 79.7 \\
\hline & on & 51600114 & PHT-S & 15-Apr.-1997 & 03:59 & & & & \\
\hline & off & 51801215 & PHT-P & 17-Apr.-1997 & $13: 27$ & & & & \\
\hline & off & 51801216 & PHT-S & 17-Apr.-1997 & $13: 42$ & & & & \\
\hline \multirow[t]{3}{*}{ (511) Davida } & on & 46502035 & PHT-P & 23-Feb.-1997 & $23: 20$ & 2.5953 & 2.5005 & 22.3 & 84.3 \\
\hline & on & 46502036 & PHT-S & 23-Feb.-1997 & $23: 35$ & & & & \\
\hline & on & 46602237 & PHT-P & 24-Feb.-1997 & $23: 13$ & & & & \\
\hline \multirow[t]{3}{*}{ (914) Palisana } & on & 71002224 & PHT-P & 26-Oct.-1997 & $05: 48$ & 2.7881 & 2.2624 & 19.3 & 111.7 \\
\hline & on & 71002225 & PHT-S & 26-Oct.-1997 & 06:03 & & & & \\
\hline & off & 71102126 & PHT-P & 27-Oct.-1997 & $08: 10$ & 2.7898 & 2.2498 & 19.2 & 112.8 \\
\hline
\end{tabular}

It is widely accepted that a particular zone of the outer main belt is characterized by objects which have been aqueously altered (Vilas et al. 1984; Barucci et al. 1998): this should be indicative of the presence of water ice in the original bodies that have been heated during the primordial phases of the solar system formation.

Spectroscopic investigation of low and moderate albedo asteroids is an essential tool to investigate their surface composition and the infrared range is certainly the most promising. The Infrared Space Observatory (ISO), an ESA project launched in November 1995 and operative until April 1998 (Kessler et al. 1996), gave the unique opportunity to observe asteroids in the unexplored range of the mid-infrared. Several ISO results on asteroids have been already presented and discussed (e.g. Dotto et al. 2000; Barucci et al. 2002; Müller \& Lagerros 2002). Here we present the results obtained for a sample of five asteroids, selected among the brightest low and moderate albedo objects at the time of ISO observations.

\section{Observations and data reduction}

The ISO observations presented here have been carried out between February and October 1997 with two different detectors of the instrument ISOPHOT: PHT-P and PHT-S.

In Table 1 the observational aspect data of the observed asteroids are listed. The background measurements (indicated in the Table as "off") have been carried out at the same sky position, but 1 or 2 days later when the asteroid was outside the field of view. Davida and Palisana have no dedicated background measurements of the PHT-S observations. The second PHT-P Davida measurement was intended to be taken off-source, but by error done again on-source.

\subsection{PHT-P data}

Multi-filter photometry was carried by PHT-P with all 3 detector subsystems at wavelengths of $10,12,25$ and $60 \mu \mathrm{m}$. The apertures used were $23^{\prime \prime}$ diameter (10 and $\left.12 \mu \mathrm{m}\right), 52^{\prime \prime}(25 \mu \mathrm{m})$ and $99^{\prime \prime}(60 \mu \mathrm{m})$. The integration times were: $64 \mathrm{~s}$ at $10 \mu \mathrm{m}$ and $32 \mathrm{~s}$ at $12 \mu \mathrm{m}$ (P1-detector); $32 \mathrm{~s}$ at $25 \mu \mathrm{m}$ (P2-detector); $32 \mathrm{~s}$ at $60 \mu \mathrm{m}$ (P3-detector).

ISOPHOT Interactive Analysis (PIA ${ }^{1}$ ) V9.0.1(e) was used for the standard data reduction up to signal level, including linear ramp fitting, deglitching on ramp and signal level and orbit dependent dark signal subtraction (Gabriel et al. 1997). Unfortunatly the integration time was in many cases not long enough to obtain stabilized signals. In these cases (P1-detector, 10 and $12 \mu \mathrm{m})$ the internal reference measurements were replaced by the "default" detector responsivity. Additionally the used non-default apertures caused problems in terms of absolute calibration of the background photometry (in comparison with COBE-DIRBE values by Hauser et al. 1998). For these reasons, all background measurements were replaced by COBE-DIRBE values (weekly maps, color corrected, PHT-P aperture size corrected).

${ }^{1}$ PIA is a joint development by the ESA Astrophysics Division and the ISOPHOT consortium. 
All source measurements have been background subtracted and color corrected. The following color correction values have been used: $10 \mu \mathrm{m}: 0.99 ; 12 \mu \mathrm{m}: 0.91 ; 25$ and $60 \mu \mathrm{m}: 1.07$. These values correspond to temperatures between 200 and $300 \mathrm{~K}$.

The analysis of similar measurements on calibration targets in the same apertures revealed that the internal calibration source of ISOPHOT (the Fine Calibration Source, FCS) leads in some cases to a flux overestimation up to $20 \%$ (P2-detector, $25 \mu \mathrm{m}$ ) and a flux underestimation up to $20 \%$ (P3-detector, $60 \mu \mathrm{m})$. This is reflected in the asymmetric uncertainties in the values. In case of strong signal transients the error values have been increased accordingly.

\subsection{PHT-S data}

PHT-S, which consists of two low-resolution grating spectrometers, covered the wavelength ranges $2.5-4.9 \mu \mathrm{m}$ (PHTSS) and 5.8-11.6 $\mu \mathrm{m}$ (PHT-SL). The obtained spectra have a resolution $\lambda / \Delta \lambda$ of about 85 for PHT-SS and of about 95 for PHT-SL. All PHT-S observations have been performed in staring mode with a default of $32 \mathrm{~s}$ dark exposure at the beginning of the measurement. The integration time was of $1024 \mathrm{~s}$ for 77 Frigga, 114 Kassandra, 308 Polyxo, 914 Palisana, and $512 \mathrm{~s}$ for 511 Davida.

PIA V8.2(e) was used for the standard data reduction up to signal level, including linear ramp fitting, deglitching on ramp and signal level and orbit dependent dark signal subtraction (Gabriel et al. 1997). The flux calibration is done with the $d y$ namic response method (Laureijs et al. 2002) by taking the ratio between the signals from the target and the calibrators along the observing time and then deriving the mean and median values, which are scaled by the known flux from the chosen calibrators. The dark current subtraction was performed before the dynamic calibration application.

The on-source measurements have been background subtracted in the following way: 1) cross check of the absolute background level with COBE-DIRBE data (weekly maps, color corrected, PHT-S beam size corrected) for the 3 dedicated background spectra (see Fig. 1);2) fit of a blackbody curve to the observed background spectra (blackbody temperatures taken from Ábrahám et al. 1999); 3) for Davida and Palisana the blackbody fit was done directly to the COBE-DIRBE values since no dedicated background measurements exist; 4) subtraction of the blackbody fits from the on-source measurements. This procedure avoids the noise introduction from the background subtraction. Figure 1 shows the PHT-S background spectra of Frigga, Kassandra, and Polyxo together with the fitted blackbody curve and the corresponding COBE-DIRBE values.

The uncertainties from the signal processing by PIA are $3-5 \%$, which reflect also the relative accuracy from pixel to pixel. The absolute uncertainty is generally stated better than $30 \%$ (Klaas et al. 1997). Since we used an interactive analysis with improved calibration we believe that the absolute calibration is better than $15 \%$ for Frigga, Kassandra and Palisana and better then $10 \%$ for Polyxo and Davida. This is also in agreement with our cross-calibration experiences between
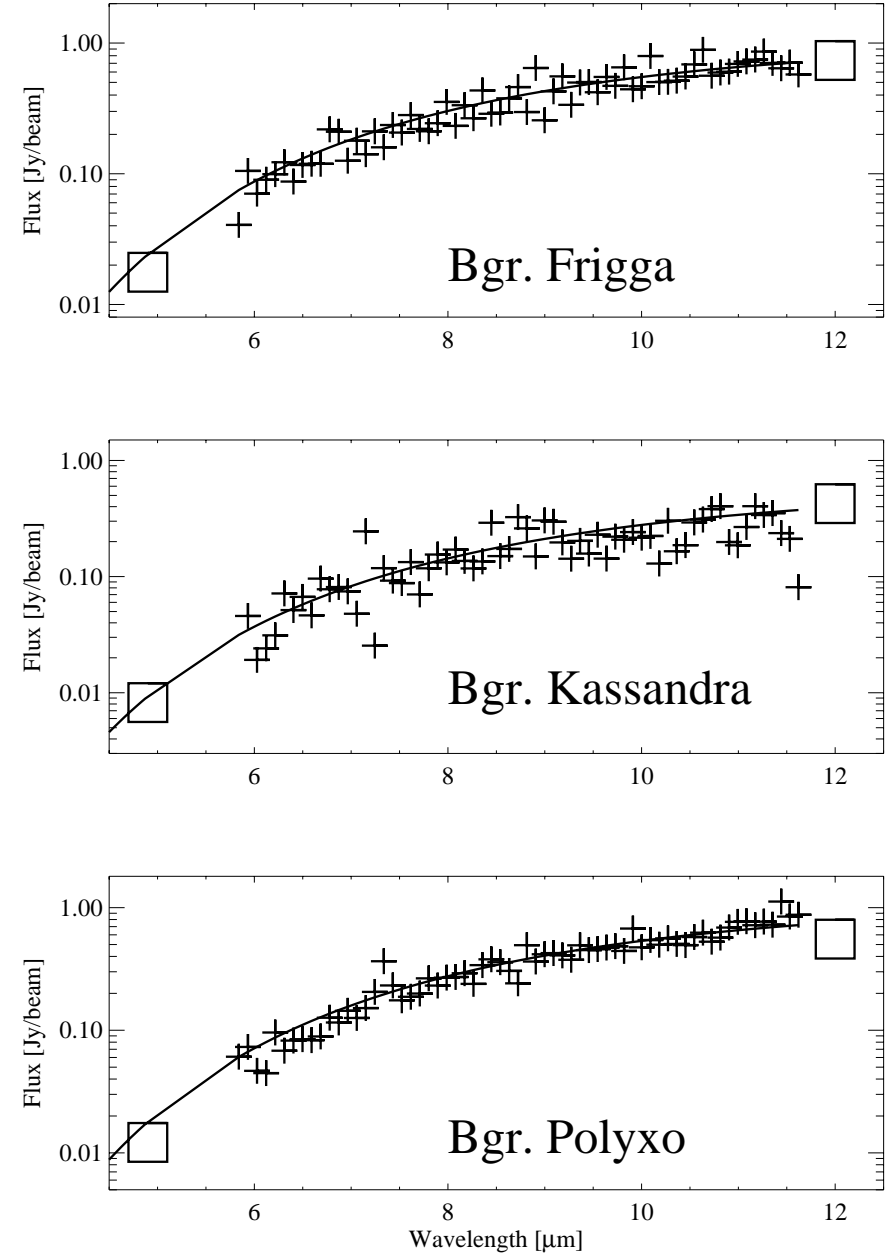

Fig. 1. PHT-S background measurements at the positions of the asteroids Frigga, Kassandra and Polyxo. The fitted blackbody curve (solid line) and the corresponding COBE-DIRBE value (box) are also shown.

different ISO instruments and with stellar models. The PHT-SS $2.5-4.9 \mu \mathrm{m}$ part of the asteroid spectra is at a very low signal level, close to the detection limit. Therefore we exclude in this paper this part of the spectrum.

In Fig. 2 the low-resolution PHT-S spectra between 5.8 and $11.6 \mu \mathrm{m}$ together with the PHT-P data are shown with the uncertainties produced by the signal processing procedure.

\section{Models}

The emission of asteroids beyond $\sim 5 \mu \mathrm{m}$ is dominated by radiation thermally emitted from their surface. A blackbody fit and the Standard Thermal Model (STM) have been applied to the obtained infrared data to determine the range of surface (blackbody and sub-solar) temperatures.

To determine the black-body temperature of the observed asteroids we fitted the infrared data with a Planck function multiplied by the solid angle of the objects. Both the solid angle and the black-body temperature were treated as free parameters.

The sub-solar temperature of the observed objects was computed by applying the STM (Lebofsky \& Spencer 1989), already used by Tedesco et al. (1992) in the IRAS asteroid 

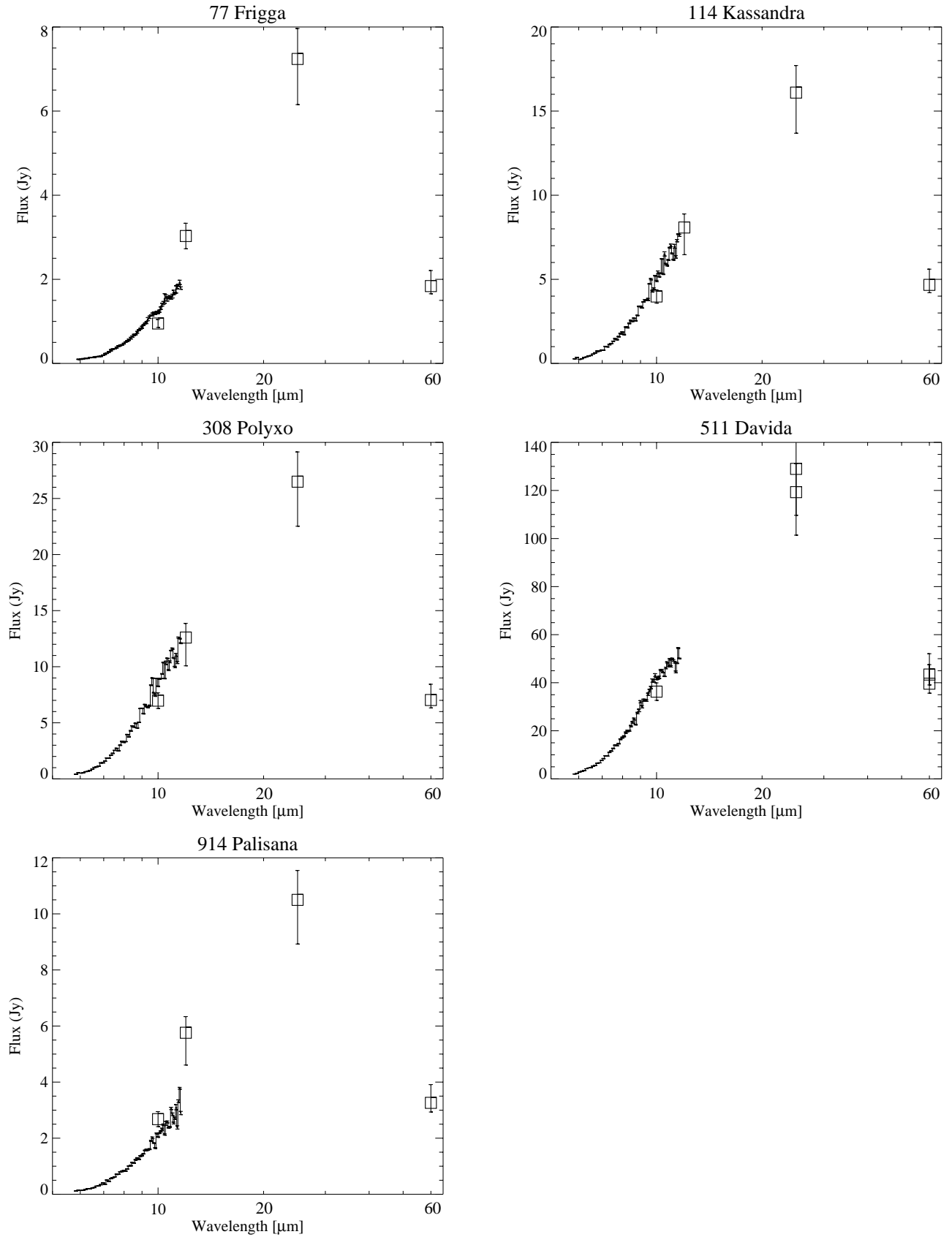

Fig. 2. PHT-S spectra between 5.8 and $11.6 \mu \mathrm{m}$ and PHT-P data (box).

survey. It assumes a non-rotating spherical asteroid, in instantaneous equilibrium with solar insolation, observed at $0^{\circ}$ solar phase angle. In this ideal situation, in which the thermal inertia is neglected, and the asteroid nightside emission is thus not taken into account, the sub-solar temperature is given as:

$T_{\mathrm{SS}}=\left[\frac{(1-A) S}{\eta \epsilon \sigma}\right]^{1 / 4}$ and $T(\Omega)=T_{\mathrm{SS}} \cos ^{1 / 4}(\Omega)$

where $\Omega$ is the solar zenith angle, $A$ is the bolometric Bond albedo, $S$ is the solar flux at the distance of the asteroid, $\eta$ (infrared beaming) is an empirical factor adjusted so that the model matches the integrated flux of the object at a given wavelength, $\epsilon$ is the wavelength-independent emissivity and $\sigma$ is the Stefan-Boltzmann constant. Moreover, the infrared beaming and the phase angle geometry are empirically corrected, generally with correction factors of $\eta=0.756$ and $0.01 \mathrm{mag} \mathrm{deg}^{-1}$, respectively (see Lebofsky \& Spencer 1989).

In Table 2 the sub-solar and black-body temperatures, and the albedo and diameter values estimated by applying STM to our data are reported. The table shows also the absolute magnitude $H$ and the slope parameter $G$ used as input, and diameters and albedos obtained by Tedesco et al. (1992) on the basis of IRAS data. Our estimations of diameters and albedos are in agreement with the values given by Tedesco et al. (1992), with the exception of the albedos of 511 Davida and 914 Palisana which deviate from the IRAS results. This can be due to differences in the viewing geometry between the IRAS and ISO observations. In the simplified STM the real 
shape of the asteroid is not taken into account: this can influence the albedo and diameter calculations. To model the thermal continuum of previous ISO observations, Dotto et al. (2000) and Barucci et al. (2002) used the advanced thermophysical model (TPM) developed by Lagerros (1996, 1997, 1998). To properly apply this advanced model we need the knowledge of several physical parameters of the analysed asteroids. Unfortunately, we do not have a good estimation of the pole direction, shape, infrared beaming and thermal inertia of the five asteroids here discussed. A comparison between the diameter and albedo values obtained by STM and TPM has been possible only for 77 Frigga, for which an estimation of the rotational state is available (Erikson 2000). We applied TPM, considering the wavelength dependent emissivity as stated by Müller \& Lagerros (1998), and default values for thermal inertia and beaming parameters $\rho$ and $f$, and we obtained results similar to those computed by STM. Since the rotational and physical parameters of the asteroids here discussed are not sufficiently well known, we preferred to apply the simplest STM, with the minimum number of free parameters, without introducing new possible sources of error.

Using STM we computed the expected flux at the time of ISO observations. Then we divided the observed spectra for the STM expected flux, obtaining the "Relative Obs/Mod".

\section{Laboratory spectra and observational results}

In order to interpret the obtained spectral behaviours we compared the observational results with the whole sample of mineral and meteorite emissivities available in the literature (Salisbury et al. 1991a,b, ASTER spectral library on http://speclib.jpl.nasa.gov). Moreover, new laboratory spectra of a few selected samples at different grain dimensions, have been obtained with the Capodimonte Observatory Bruker IFS66v interferometer, following the procedures already described in Dotto et al. (2000) and Barucci et al. (2002). Since asteroid spectra seem to be dominated by the effect of fine particles (Le Bertre \& Zellner 1980), all the laboratory samples we considered are particulate.

In Figs. 3-7 we plot the "Relative Obs/Mod" obtained for each observed asteroid, compared with the emissivities of some mineral and/or meteorite analogs. In the considered wavelength range the most diagnostic feature is the Christiansen peak which is associated with the principal molecular vibration band, where the refractive index changes rapidly, and occurs at a wavelength that for silicates is just short of the $\mathrm{Si}-\mathrm{O}$ stretching vibration bands. This feature, directly related to the mineralogy and the grain size, appears generally as a peak between 7.5 and $9.5 \mu \mathrm{m}$.

77 Frigga. The taxonomic classification of this object is still an open problem. It has been classified as MU-type (Tholen 1989), D2-type (Barucci et al. 1987), and Xe (Bus \& Binzel 2002).

On the basis of our ISO data we obtained a diameter of $70 \pm 4 \mathrm{~km}$ and an albedo of $0.146 \pm 0.005$, in close agreement with the previous IRAS determinations. These values have been computed using STM on PHT-S and PHT-P data,

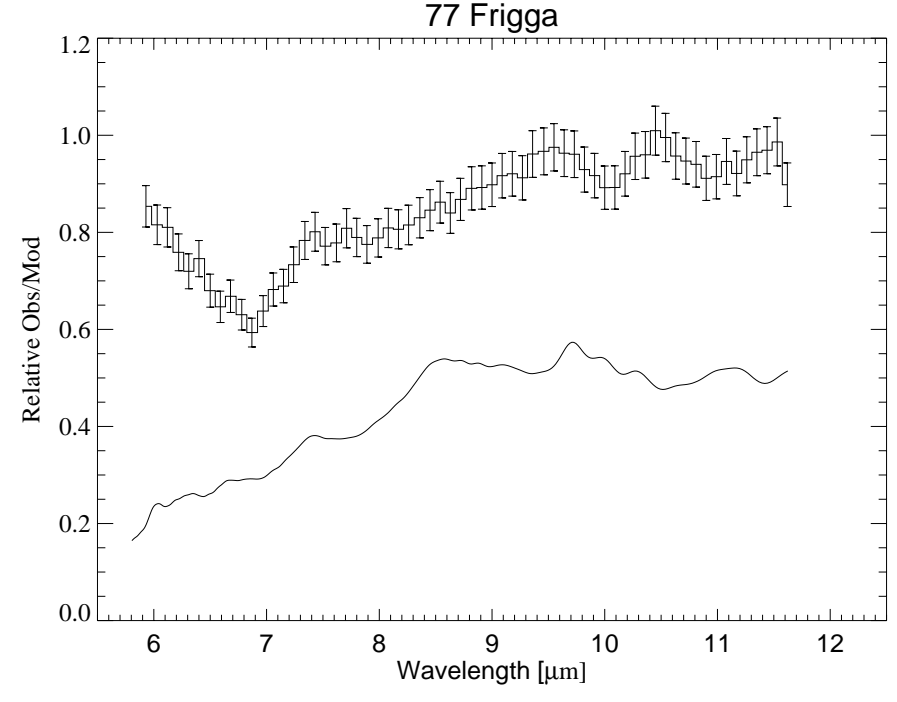

Fig. 3. Relative PHT-S Obs/Mod of 77 Frigga compared with the winonaite meteorite Winona (continuous line). The spectrum of Winona is vertically offset for clarity.

eliminating the observation at $25 \mu \mathrm{m}$ which gives an overestimation of about $40 \%$ in the diameter.

Figure 3 shows the emissivity obtained by dividing the PHT-S data by the STM expected flux. The comparison of this spectrum with the whole sample of available meteorite and mineral emissivities was unsuccessfull. The only possible analogy, even if very weak, seems to be with the emissivity of the Winona meteorite sample belonging to the ASTER database. Winona belongs to the winonaite meteorites, a group of primitive achondrites associated with the IAB/IIICD iron meteorites. Its structure is characterized by silicate inclusions in IAB irons. Although this is just a tentative interpretation of the obtained ISO mid-infrared spectrum of Frigga, the silicated iron nature of the meteorite Winona seems to be in agreement with the debated classification of this asteroid.

114 Kassandra and 308 Polyxo. Asteroids 114 Kassandra and 308 Polyxo have been classified as belonging to the T class by Tholen (1989), as a D2-type object by Barucci et al. (1987), and as Xk and T, respectively, by Bus \& Binzel (2002). On the basis of ISO PHT-P and PHT-S data we obtained diameter and albedo values which are in agreement (to within 10\%) with the previous determinations obtained on the basis of the IRAS data.

Britt et al. (1992) showed that 114 Kassandra has a 0.4$2.5 \mu \mathrm{m}$ spectrum very similar to troilite, while 308 Polyxo shows in the same wavelength range a completely different spectral behavior. In the mid-infrared range, on the contrary, these two objects show a spectral behavior similar to that one of Ornans, a $\mathrm{CO} 3$ carbonaceous chondrite meteorite. Figures 4 and 5 show the comparison between the emissivity of Kassandra and Polyxo and the laboratory emissivity of an Ornans sample at grain sizes between 0 and $20 \mu \mathrm{m}$. The behaviour of the spectrum around $9.2 \mu \mathrm{m}$ seems to be consistent with the Christiansen peak of the Ornans sample. This result is surprising since Ornans is a type 3 carbonaceous meteorite chondrite (CO3) which seems to show the presence of 
Table 2. Absolute magnitude $H$, slope parameter $G$, computed temperatures and diameter and albedo values, IRAS diameters and albedos $(D$ and $\left.p_{H}\right)$ with their uncertainties $\left(\sigma_{D}\right.$ and $\left.\sigma p_{H}\right)$.

\begin{tabular}{|c|c|c|c|c|c|c|c|c|c|c|}
\hline Object & $\begin{array}{c}H \\
\text { (mag) }\end{array}$ & $G$ & $\begin{array}{c}\text { Diameter } \\
(\mathrm{km})\end{array}$ & Albedo & $\begin{array}{c}\text { Black-body } \\
\text { temp. } \\
(\mathrm{K})\end{array}$ & $\begin{array}{c}\text { Sub-solar } \\
\text { temp. } \\
(\mathrm{K})\end{array}$ & $\begin{array}{c}\text { IRAS } \\
D \\
(\mathrm{~km})\end{array}$ & $\begin{array}{c}\text { IRAS } \\
\sigma_{D} \\
(\mathrm{~km})\end{array}$ & $\begin{array}{c}\text { IRAS } \\
p_{H}\end{array}$ & $\begin{array}{l}\text { IRAS } \\
\sigma p_{H}\end{array}$ \\
\hline 77 Frigga & 8.52 & 0.16 & $70 \pm 4$ & $0.146 \pm 0.005$ & 223 & 259 & 69.25 & 2.1 & 0.144 & 0.009 \\
\hline 114 Kassandra & 8.26 & 0.15 & $103 \pm 4$ & $0.084 \pm 0.005$ & 228 & 265 & 99.64 & 1.9 & 0.0884 & 0.003 \\
\hline 308 Polyxo & 8.17 & 0.21 & $151 \pm 7$ & $0.043 \pm 0.002$ & 232 & 265 & 140.69 & 3.8 & 0.0482 & 0.003 \\
\hline 511 Davida & 6.22 & 0.16 & $303 \pm 8$ & $0.064 \pm 0.003$ & 232 & 268 & 326.07 & 5.3 & 0.054 & 0.0023 \\
\hline 914 Palisana & 8.76 & 0.15 & $71 \pm 7$ & $0.113 \pm 0.004$ & 220 & 257 & 76.61 & 1.7 & 0.0943 & 0.004 \\
\hline
\end{tabular}

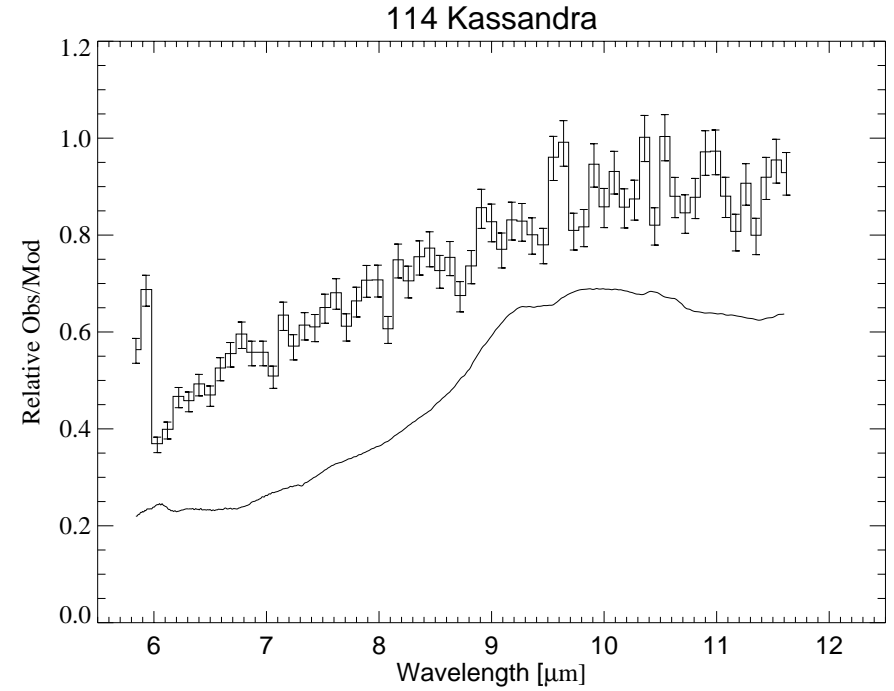

Fig. 4. Relative PHT-S Obs/Mod of 114 Kassandra compared with the $\mathrm{CO} 3$ carbonaceous chondrite meteorite Ornans (continuous line). The spectrum of Ornans is vertically offset for clarity.

aqueous alteration processes (Zolensky \& McSween 1988). 308 Polyxo has been shown to have a strong feature of water of hydration feature at $3.0 \mu \mathrm{m}$ while in the spectrum of 114 Kassandra this feature is completely absent (Jones et al. 1990). These differences in the interpretation of the surface composition can be due to possible variations with different rotational phases. Further observations at near-infrared wavelength of these two asteroids are needed in order to investigate the possibility that these objects have really undergone some aqueous alteration processes.

511 Davida. Asteroid 511 Davida is the fifth biggest asteroid with an IRAS diameter of $326 \mathrm{~km}$. For this object PHT-P obtained multi-filter photometric data only at 10,25 , and $60 \mu \mathrm{m}$. On the basis of our complete ISO data sample we computed a diameter of $303 \mathrm{~km}$ and an albedo of 0.064 .

Davida as been classified by Tholen (1989), Barucci et al. (1987) and Bus \& Binzel (2002) as belonging to the C class. Jones et al. (1990) detected hydrated silicates (via 3- $\mu \mathrm{m}$ spectrometry) on the surface of this asteroids, while Hiroi et al. (1996) found a good match between the 0.3-3.6 $\mu \mathrm{m}$ spectrum

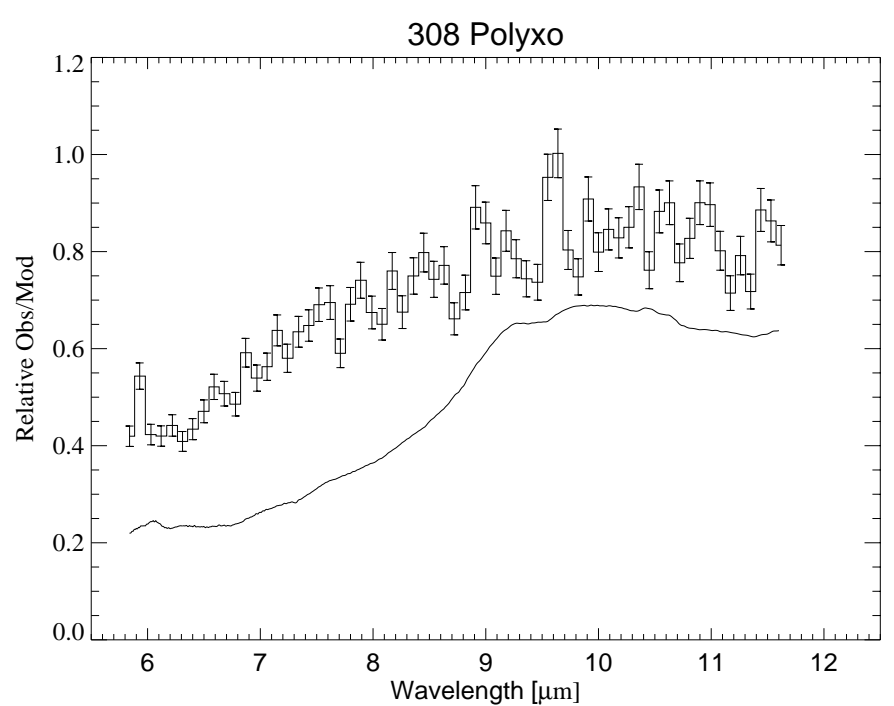

Fig. 5. Relative PHT-S Obs/Mod of 308 Polyxo compared with the $\mathrm{CO} 3$ carbonaceous chondrite meteorite Ornans (continuous line). The spectrum of Ornans is vertically offset for clarity.

of Davida and that one of the unusual CI/CM meteorite B-7904. On the basis of the analysis of heated Murchison samples they inferred that B-7904 has probably been heated up to $500-600{ }^{\circ} \mathrm{C}$.

Figure 6 shows the emissivity of Davida between 5.8 and $11.6 \mu \mathrm{m}$ which is consistent with that one of carbonaceous chondrites as reported by Salisbury et al. (1991a). In particular the spectral behaviour between 6.5 and $11.5 \mu \mathrm{m}$ suggests the comparison with the emissivity of the CM-type carbonaceous chondrite meteorite Murchison given by the ASTER database. This result seems to confirm that Davida is a large body which suffered aqueous alteration processes.

914 Palisana. Asteroid 914 Palisana is classified as CU-type by Tholen (1989) and as a D3-type by Barucci et al. (1987). On the basis of our ISO data we computed a diameter of $71 \mathrm{~km}$ and an albedo of 0.113. Also in this case the PHT-P measurement at $25 \mu \mathrm{m}$ gives an overestimation of diameter and albedo of more than $30 \%$. 


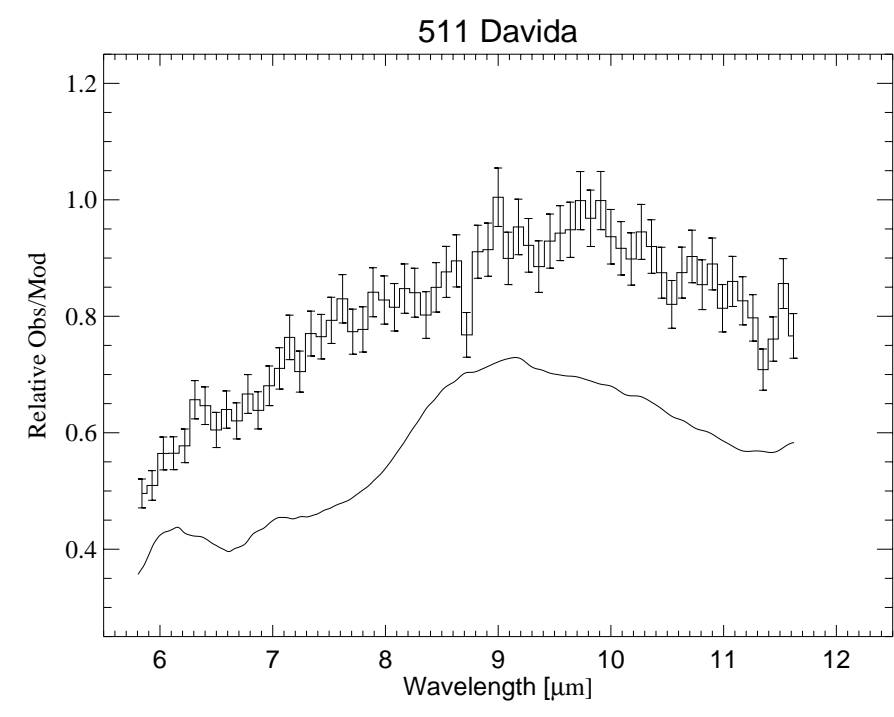

Fig. 6. Relative PHT-S Obs/Mod of 511 Davida compared with the CM-type carbonaceous chondrite meteorite Murchison (continuous line). The spectrum of Murchison is vertically offset for clarity.

Fitzsimmons et al. (1994) showed that Palisana exhibits in the visible range large-scale absorption characteristic of phyllosilicates.

The spectral behavior, shown in Fig. 7 , is after $7 \mu \mathrm{m}$ flat and featureless. We tried to compare this object with many mineral and meteorite emissivities. In particular we compared the obtained spectrum with the emissivity of phyllosilicates and $\mathrm{CI} / \mathrm{CM}$ carbonaceous chondrite meteorites which are related to aqueous alteration products. None of the available laboratory spectra of minerals and meteorites matched the observed spectrum. As an example we report in Fig. 7 the emissivities of a sample of the CI-type meteorite Orgueil at grain sizes between 0 and $50 \mu \mathrm{m}$ and a sample of powdered kaolinite (phyllosilicate), obtained by laboratory experiments at the Capodimonte Observatory.

\section{Conclusions}

In this paper we present the first results on mid-infrared wavelength range of five asteroids. ISO gave the unique opportunity to observe asteroids at this wavelength range and we obtained photometric data up to $60 \mu \mathrm{m}$ and low resolution spectra between 5.8 and $11.6 \mu \mathrm{m}$. On the basis of these data we computed albedos and diameters of the observed asteroids. The obtained results are in agreement with the estimations computed on the basis of the IRAS data.

The interpretation of the obtained spectra is neither easy nor unique. A tentative interpretation has been suggested by comparing the observed spectral behaviours with the emissivities of meteorites which are our best available analogs of asteroidal surface materials. The theories about the thermal history of C-type asteroids seems to be confirmed by the match between the spectrum of 511 Davida and the emissivity of the CM-type carbonaceous chondrite meteorite Murchison. While the tentative analogy between the spectrum of Frigga and the emissivity

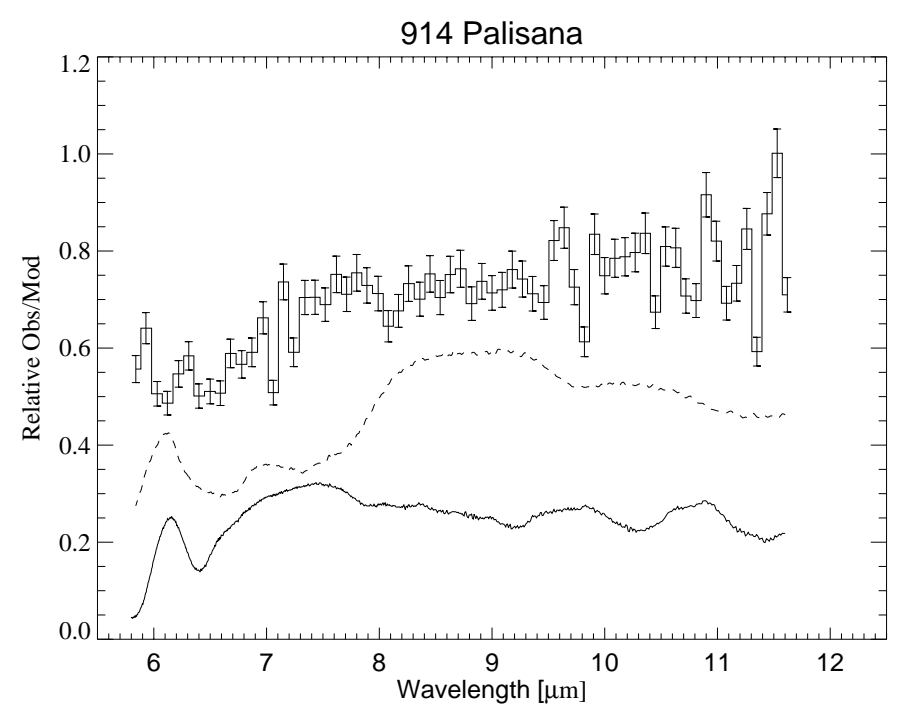

Fig. 7. Relative PHT-S Obs/Mod of 914 Palisana compared with the CI-type carbonaceous chondrite meteorite Orgueil (dashed line) and a phyllosilicate kaolinite (continuous line). The spectra of Orgueil and kaolinite are vertically offset for clarity.

of Winona is consistent with the still debated taxonomic classification of this asteroid.

Problems relative to the calibration of these faint objects due to non-standard instrument configurations have affected the quality of the ISO data, especially the point at $25 \mu \mathrm{m}$. For this reason and due to the difficulty in interpreting the surface spectra, the obtained results and the suggested interpretations cannot be conclusive. This work can be considered a support for future observations of asteroids in these wavelengths by space platform projects, planned to be operative in next future (e.g. the Space Infrared Telescope Facility SIRTF).

Acknowledgements. J.R.B., V.M., and L.C. whish to thank ASI and MIUR for supporting the experimental work.

\section{References}

Ábrahám, P., Leinert, C., Acosta-Pulido, J. A., et al. 1999, ESA SP-427, ed. P. Cox, \& M. F. Kessler, 145

Barucci, M. A., Capria, M. T., Coradini, A., et al. 1987, Icarus, 72, 304

Barucci, M. A., Doressoundiram, A., Fulchignoni, M., et al. 1998, Icarus, 132,388

Barucci, M. A., Dotto, E., Brucato, J. R., et al. 2002, Icarus, 156, 202

Britt, D. T., Bell, J. F., Haack, H., et al. 1992, Meteoritics, 27, 207

Bus, B. J., \& Binzel, R. P. 2002, Icarus, 158, 146

Dotto, E., Müller, T. G., Barucci, M. A., et al. 2000, A\&A, 358, 1133

Erikson, A. 2000, Ph.D. Thesis, Freie Universität, Berlin, Germany

Fitzsimmons, A., Dahlgren, M., Lagerkvist, C.-I., et al. 1994, A\&A, 282,634

Gabriel, C., Acosta-Pulido, J. A., Heinrichsen, I., et al. 1997, in ASP Conf. Ser. 125, ed. G. Hunt \& H. Payne, 108

Gradie, J. C., Chapman, C. R. \& Tedesco, E. F. 1989, in Asteroids II (University of Arizona Press, Tucson) 316

Hauser, M. G., Kelsall, T., Leisawitz, D., et al. 1988, COBE Ref. Pub. No. 98-A (Greenbelt, MD: NASA/GSFC), available in electronic form from the NSSDC 
Hiroi, T., Zolensky, M. E., Pieters, C. M., et al. 1996, Meteoritics \& Plan. Sci., 31, 321

Jones, T. D., Lebofsky, L. A., Lewis, J. S., et al. 1990, Icarus, 88, 172 Kessler, M. F., Steinz, J. A., Anderegg, M. E., et al. 1996, A\&A, 315, L27

Klaas, U., Acosta-Pulido, J. A., Ábrahám, P., et al. 1997, ESA SP-419, 113

Lagerros, J. S. V. 1996, A\&A, 310, 1011

Lagerros, J. S. V. 1997, A\&A, 325, 1226

Lagerros, J. S. V. 1998, A\&A, 332, 1123

Laureijs, R. J., Klaas, U., Richards, P. J., et al. 2002, The ISO Handbook, Volume IV: PHT - The Imaging Photo-Polarimeter, SAI-99-069/Dc, Version 2.0

Le Bertre, T., \& Zellner, B. 1980, Icarus, 43, 172

Lebofsky, L. A., \& Spencer, J. R. 1989, in Asteroids II (University of Arizona Press, Tucson), 128
Müller, T. G., \& Lagerros, J. S. V. 1998, A\&A, 338, 340

Müller, T. G., \& Lagerros, J. S. V. 2002, A\&A, 381, 324

Salisbury, J. W., D’Aria, D. M., \& Jarosewichi, E. 1991a, Icarus, 92, 280

Salisbury, J. W., Walter, L. S., Vergo, N., et al. 1991b, Infrared (2.1-25 $\mu \mathrm{m}$ ) spectra of minerals, (Baltimore: Johns Hopkins Univ. Press)

Tedesco, E. F., Veeder, G. J., Fowler, J. W., et al. 1992, The IRAS Minor Planet Survey (Phillips Laboratory)

Tholen, D. 1989, in Asteroids II (University of Arizona Press, Tucson), 1139

Tholen, D., \& Barucci, M. A. 1989, in Asteroids II (University of Arizona Press, Tucson), 298

Vilas, F., Jarvis, K. S., \& Gaffey, M. J. 1994, Icarus, 109, 274

Zolensky, M., \& McSween, H. Y. 1988, in Meteorites (University of Arizona Press, Tucson), 114 